\title{
Influence of knee osteoarthritis and aging on isokinetic muscle strength of the lower limbs in women
}

\author{
Yuliang Sun ${ }^{1, a}$ \\ ${ }^{1}$ School of Physical Education, Shaanxi Normal University, Shaanxi 710119, China; \\ asunsyl123@163.com
}

Keywords: osteoarthritis, knee joint, the elderly, muscle strength.

\begin{abstract}
Aging is a multifactorial process that results in changes in the skeletal muscle then leads to decreased levels of muscle strength. Knee osteoarthritis (OA) is one of the most predominant causes of pain, functional decline and disability in the elderly population worldwide. In China the prevalence of radiographic knee OA was $42.8 \%$ in older women and $21.5 \%$ in older men. It has been shown to affect a multitude of Isokinetic muscle strength parameters. This study sought to investigate the different impacts of knee osteoarthritis and aging on isokinetic muscle strength of the lower limbs in women. We found that knee osteoarthritis might have a further bad influence on knee and hip joint of elderly women and they also had a significantly higher flexor-to-extensor moment ratios (f/e) than older controls. Reduced muscular strength emerge as a result of aging, isokinetic muscle strength decrease of lower limbs in KOA patients are more pronounced compared to older controls, especially in knee.
\end{abstract}

\section{Introduction}

Aging is a multifactorial process that results in changes in the skeletal muscle then leads to decreased levels of muscle strength [1]. According to previous studies, muscle strength starts to diminish early in the life span, with losses of about $12-15 \%$ per decade after 50 years of age [2].

In addition, Osteoarthritis (OA), the most prevalent form of arthritis, affects an increasing proportion of the population [3], with the knee being the most commonly affected joint in the lower limb[4]. About $6 \%$ of Asian males and 12\% of Asian females suffer from knee OA[5]. Knee OA is one of the most predominant causes of pain, functional decline and disability in the elderly population worldwide. Patients with knee OA tend to avoid physical activity in order to prevent pain. Muscles around the knee are thought to not only produce movement, but increase joint stability and reduced joint loading which is important for patients with knee OA [6-7]. However, inactivity leads to deterioration of muscle strength and consequently to more limitations in physical activities in knee OA patients [8]. According to a 2007 report from WHO [9], movements become limited in $80 \%$ of OA patients older than 60 years and 25\% cannot perform major daily activities of life.

Isokinetic is a reference method for evaluation of muscle strength. It enables a functional diagnosis specifying levels of disability and performance deficits in the subject knee OA.

This study sought to investigate the different impacts of knee osteoarthritis and aging on isokinetic muscle strength of the lower limbs in women and then supply biomechanics theoretical basis for the training of lower extremity muscle strength in patients with knee osteoarthritis.

\section{Methods}

All the tests were completed in Shanghai University of Sport, Sports Performance Research Centre (Shanghai, People's Republic of China). The eight testers in this study were graduate students who majored in biomechanics and had at least 1 year of training with the equipment and techniques used in this study. Isokinetic extensor and flexor strength of hip, knee and ankle were collected in 22 knee osteoarthritis participants (mean age: 63.71 \pm 3.02 ) with radiographic symptom Kellgren-Lawrence 
grade 2/3 (KLG), 15 older healthy controls (mean age: 62.47 \pm 2.56 ) and 10 younger healthy controls (mean age: 26.20 \pm 2.30 ). This study was approved by the local ethical committee of Shanghai University of Sport, and all participants gave informed written consent prior to testing.

Isokinetic muscle strength of knee, hip and ankle were measured with an isokinetic dynamometer (Contrex , CMV AG, Switzerland). The maximal isokinetic torque was determined in one set of five maximal repetitions, and the highest peak torque was used in the data analysis. There was a 5-minute rest after each maximal isokinetic torque test to avoid possible fatigue effects. In addition, the participants were encouraged verbally to exert as much force as possible. All testing positions were based on the previous studies showing good to very-good between trial and between-day reliability of a dynamometer for the lower extremity strength measurement in similar populations[10]. The more symptomatic knee was used for the OA subjects while the dominant leg defined by kicking preference was evaluated for the younger and older healthy controls. The average muscle strength [normalized to body weight (BW)] was used in the analyses.

Statistical analysis will be carried out using SPSS 20.0 and microsoft office excel. One-way analysis of variance (ANOVA) was used to evaluate differences in demographic, and strength variables between $\mathrm{HY}$ and $\mathrm{HO}, \mathrm{HO}$ and $\mathrm{OA}$. All data are expressed as mean \pm standard deviation (SD). A conservative level of significance is set at $\mathrm{P}<0.05$.

\section{Results and Discussion}

Concentric peak torques (PT) of flexor muscle and extensor muscle of knee and ankle in older controls were less than that in younger controls $(\mathrm{P}<0.01)$. However, there was no significant difference between the PT of flexor muscle and extensor muscle of hip in older controls and that in younger controls. These finding indicated that age-related changes occurred in the musculoskeletal system and central nervous system (CNS), which are believed to be associated with muscle strength decline in older people. The high-light is that the flexor muscles and extensor muscles about the knee and ankle joints are more sensitive to these changes.

Comparing with elderly controls, OA patients were observed of worse extensor muscle PT in knee and flexor / extensor muscle PT in hip $(\mathrm{P}<0.05)$, but similar observation were not found in ankle. That is to say, knee osteoarthritis might have a further bad influence on knee and hip joint of elderly women (Table 1). That is Osteoarthritis could significantly affect the strength of the lower extremity, especially at the knee and hip joints.

Table 1 Concentric peak torques (PT) of flexor muscle and extensor muscle of hip, knee and ankle in HY, HO and OA groups

\begin{tabular}{ccccccc}
\hline & \multicolumn{2}{c}{$\mathrm{HY}(\mathrm{n}=10)$} & \multicolumn{2}{c}{$\mathrm{HO}(\mathrm{n}=15)$} & \multicolumn{2}{c}{$\mathrm{OA}(\mathrm{n}=22)$} \\
\cline { 2 - 7 } & $\begin{array}{c}\mathrm{PTe} \\
(\mathrm{Nm} / \mathrm{kg})\end{array}$ & $\begin{array}{c}\mathrm{PTf} \\
(\mathrm{Nm} / \mathrm{kg})\end{array}$ & $\begin{array}{c}\mathrm{PTe} \\
(\mathrm{Nm} / \mathrm{kg})\end{array}$ & $\begin{array}{c}\mathrm{PTf} \\
(\mathrm{Nm} / \mathrm{kg})\end{array}$ & $\begin{array}{c}\mathrm{PTe} \\
(\mathrm{Nm} / \mathrm{kg})\end{array}$ & $\begin{array}{c}\mathrm{PTf} \\
(\mathrm{Nm} / \mathrm{kg})\end{array}$ \\
\hline Hip & $1.10 \pm 0.74$ & $-0.85 \pm 0.2$ & $1.13 \pm 0.37$ & $-0.89 \pm 0.20$ & $0.88 \pm 0.30$ & $-0.52 \pm 0.17 \dagger \dagger$ \\
& & 1 & & & $\dagger$ & \\
Knee & $1.97 \pm 0.54$ & $-1.55 \pm 0.4$ & $1.10 \pm 0.24^{*}$ & $-0.62 \pm 0.18^{*}$ & $0.78 \pm 0.44$ & $-0.76 \pm 0.43$ \\
& & 1 & $*$ & $*$ & $\dagger$ & \\
Ankle & $0.97 \pm 0.92$ & $-0.93 \pm 0.3$ & $0.17 \pm 0.04^{*}$ & $-0.53 \pm 0.18^{*}$ & $0.18 \pm 0.05$ & $-0.43 \pm 0.12$ \\
& & 2 & & & & \\
\hline
\end{tabular}

Table 1. Mean \pm standard deviation; $\mathrm{n}=$ number of participants; $\mathrm{HY}=$ healthy younger controls; $\mathrm{HO}=$ healthy older controls; $\mathrm{OA}=$ osteoarthritis patients; * group $\mathrm{HO}$ vs. group $\mathrm{HY},{ }^{*} \mathrm{p}<0.05,{ }^{* *} \mathrm{p}<0.001 ; \dagger$ group OA vs. group $\mathrm{HO}, \dagger \mathrm{p}<0.05$, $\dagger \dagger \mathrm{p}<0.001$; $\mathrm{PTe}=$ peak torques of extensor muscle; $\mathrm{PTf}=$ peak torques of flexor muscle.

Analysis-of-variance showed that KOA patients had significantly higher flexor-to-extensor moment ratios $(\mathrm{f} / \mathrm{e})$ than older controls $(\mathrm{P}<0.05)$ at the knee and the hip joints. These were probably 
because of the need for better joint stability or a lower quadriceps capacity. There were also significant difference between the hip flexor-to-extensor moment ratios in the group HY and that in the group OA. Same results were found at the knee joint between these two groups. (Table 2). Our previous study has shown that decreased maximal isokinetic torque (muscle strength) was related to greater PMT and disappearance of asymmetry in older adults who were at higher risk of fall, especially in the lower limb. Therefore, the fall risk could be significantly higher for OA patients [11].

Table 2 Flexor-to-extensor moment ratios (f/e) of hip, knee and ankle in HY, HO and OA groups

\begin{tabular}{cccc}
\hline & $\mathrm{HY}(\mathrm{n}=10)$ & $\mathrm{HO}(\mathrm{n}=15)$ & $\mathrm{OA}(\mathrm{n}=22)$ \\
\cline { 2 - 4 } & $\mathrm{f} / \mathrm{e}(\%)$ & $\mathrm{f} / \mathrm{e}(\%)$ & $\mathrm{f} / \mathrm{e}(\%)$ \\
\hline Hip & $82.58 \pm 25.37$ & $83.44 \pm 14.20$ & $105.13 \pm 31.34$ \\
& & & $\dagger$ \\
Knee & $53.20 \pm 9.00$ & $54.06 \pm 7.47$ & $64.39 \pm 18.56 \dagger$ \\
Ankle & $304.01 \pm 117.8$ & $310.21 \pm 100.1$ & $250.70 \pm 82.66$ \\
& 2 & 1 & \\
\hline
\end{tabular}

Table 2. Mean \pm standard deviation; $\mathrm{n}=$ number of participants; $\mathrm{HY}=$ healthy younger controls; $\mathrm{HO}=$ healthy older controls; $\mathrm{OA}=$ osteoarthritis patients; $\uparrow$ group $\mathrm{OA}$ vs. group $\mathrm{HO}, \dagger \mathrm{p}<0.05$; f/e= flexor-to-extensor moment ratios.

\section{Conclusion}

In summary, although reduced muscular strength emerge as a result of aging, isokinetic muscle strength decrease of lower limbs in knee OA patients are more pronounced compared to older controls, especially in knee. Patients with knee OA exhibit deficits in muscle strength and while they play an important role in the self-reported function of patients with knee osteoarthritis. It is believed that age-related changes in the musculoskeletal system and central nervous system (CNS), which are believed to be associated with fall risk. On the basis of our findings, there is no doubt that the situation in knee OA population is worse. That is the knee OA patients suffers from a higher fall risk. The main limitation of this study is that it is an observational study and so causal relationship cannot be concluded, further studies are needed. Moreover, planning of the suitable physical activities among knee OA patient to improve muscle strength is necessary. Interventions, such as strength exercise or Tai Chi, are needed in future study to explore the effect of increasing maximal isokinetic torque on fall risks.

\section{Acknowledgments}

This study was supported by the Fundamental Research Funds for the Central Universities (Project No.GK201603129) and MOE (Ministry of Education in China) Project of Humanities and Social Sciences (Project No.16XJC890001).

\section{References}

[1] Seene T, Kaasik P. Muscle weakness in the elderly: role of sarcopenia, dynapenia, and possibilities for rehabilitation. Eur Rev Aging Phys Act. 2012; 9(2):109-17. http://dx.doi. org/10.1007/s11556-012-0102-8

[2] Hurley BF: Age, gender, and muscular strength. J Gerontol A Biol Sci Med Sci, 1995, 50: 41-44. [Medline] 
[3] Felson, D. T., Lawrence, R. C., Dieppe, P. A., Hirsch, R., Helmick, C. G., Jordan, J. M., ... Fries, J. F. (2000). Osteoarthritis: new insights. Part 1: the disease and its risk factors. Ann Intern Med, 133(8), 635-646.

[4] Oliveria, S. A., Felson, D. T., Reed, J. I., Cirillo, P. A., \& Walker, A. M. (1995). Incidence of symptomatic hand, hip, and knee osteoarthritis among patients in a health maintenance organization. Arthritis Rheum, 38(8), 1134-1141.

[5] Fransen, M., Bridgett, L., March, L., Hoy, D., Penserga, E., \& Brooks, P. (2011). The epidemiology of osteoarthritis in Asia. Int $\mathrm{J}$ Rheum Dis, 14(2), 113-121. doi: 10.1111/j.1756-185X.2011.01608.X

[6] Bennell, K.L.,Wrigley, T.V., Hunt, M.A., Lim, B.-W., Hinman, R.S., 2013. Update on the role of muscle in the genesis and management of knee osteoarthritis. Rheum. Dis. Clin. N. Am. 39, 145-176.

[7] Mikesky, A.E., Meyer, A., Thompson, K.L., 2000. Relationship between quadriceps strength and rate of loading during gait in women. J. Orthop. Res. 18, 171-175.

[8] Holla JF, van der Leeden M, Knol DL, Peter WF, Roorda LD, Lems WF, et al. Avoidance of activities in early symptomatic knee osteoarthritis: results from the CHECK cohort. Ann Behav Med 2012; 44:33e42.

[9] Chronic rheumatic conditions Chronic diseases and health promotion 2007 June 22, 2007 Available from: http://www.who.int/chp/topics/rheumatic/en/.

[10] Bennell, K.L., Hunt, M.A., Wrigley, T.V., Hunter, D.J., Hinman, R.S., 2007. The effects of hip muscle strengthening on knee load, pain, and function in people with knee osteoarthritis: A protocol for a randomised, single-blind controlled trial. BMC Musculoskelet. Disord. 8, 121

[11] Wang, D., Jian, Z., Sun, Y., Zhu, W., Tian, S., \& Yu, L. (2016). Evaluating the fall risk among elderly population by choice step reaction test. Clinical Interventions in Aging, Volume 11(Issue 1), 1075-1082. 